\title{
Femoral Diaphysis Reconstructed with Intercalary Endoprothesis in \\ A Case of Parosteal Osteosarcoma
}

\section{IPS Oberoi*, Abhishek, Satvir Singh, DS Solanki and Mohinder S Chib}

Orthopedics, Artemis Hospital India

*Corresponding Author: IPS Oberoi, Orthopedics, Artemis Hospital India. DOI: 10.31080/ASOR.2020.03.0142
Received: January 09, 2020

Published: January 29, 2020

(C) All rights are reserved by IPS Oberoi. et al.

\begin{abstract}
Parosteal osteosarcoma (POS) is a rare, slow-growing malignant tumour that predominantly occurs on the surface of the metaphysis of long bones. POS accounts for $\sim 1-2 \%$ of primary malignant bone tumours.

Wide resection of POS is recommended to prevent local recurrence and the rate of distant metastasis; however, the optimal technique for long bone diaphyseal reconstruction following tumour resection is undecided.

The present case report describes the treatment of a 27-year old patient diagnosed with POS localized in the diaphysis of the right femur. A intercalary endoprosthesis was used to achieve anatomical and functional reconstruction for a $10 \mathrm{~cm}$ bone defect following en bloc tumour resection.

These intercalary implants helps in early recovery of the patients that is not possible in biological method of treatment. However, long-term follow studies are required to know the longevity of these implants and complication related to them.
\end{abstract}

Keywords: Intercalary; Endoprosthesis; Osteosarcoma; Limb Salvage

\section{Abbreviations}

POS: Parosteal Osteosarcoma; MRI: Magnetic Resonance Imaging; PET: Positron Emission Tomography; LC-DCP: Limited Bone Contact Dynamic Compression Plate.

\section{Introduction}

Parosteal Osteosarcoma (POS) is a rare, slow-growing malignant tumour that predominantly occurs on the surface of the metaphysis of long bones. POS accounts for $\sim 1-2 \%$ of primary malignant bone tumours [1], 4 - 6\% of all osteosarcomas [2] and 70\% of surface osteosarcomas, with a slight female predilection and a peak incidence in the third and fourth decades of life [1]. Unlike conventional osteosarcomas, POS is typically a well differentiated low-grade lesion that has a low tendency to metastasize and has a better prognosis $[1,3,4]$.

The most commonly involved sites of POS include the distal femur, proximal tibia and proximal humerus. Approximately $90 \%$ of POS cases involve the metaphysis and $\sim 67 \%$ are confined to the metaphysis. The posterior aspect of the distal femur accounts for $\sim 70 \%$ of all cases [5], and furthermore, diaphyseal involvement is observed in $<10 \%$ of all cases [6-7].

Wide resection of POS is recommended to prevent local recurrence and the rate of distant metastasis; however, the optimal technique for long bone diaphyseal reconstruction following tumour resection is undecided. There are a number of techniques available for femoral reconstruction of diaphyseal defects following excision of bone tumours. These include the use of autogenous vascularized fibular grafts [8], segmental allografts [9], autogenous extracorporeally irradiated bone [10], distraction osteogenesis [11] and intercalary endoprostheses [12]

The present case report describes the treatment of a 27-year old patient diagnosed with POS localized in the diaphysis of the right femur. A intercalary endoprosthesis was used to achieve anatomical and functional reconstruction for a $10 \mathrm{~cm}$ bone defect following en bloc tumour resection.

\section{Case Report}

A 27 year old male presented to us with a rare parasternal sarcoma (Biopsy proven) of right femur who underwent chemotherapy in Iraq. Because of high doses of steroid during chemo he developed Avascular necrosis of left hip. He was bed ridden and in severe pain. In 1st stage we did ceramic total hip replacement to make him out of bed, our oncologist optimised chemotherapy to contain the osteosarcoma.

Physical examination the patient had full active range of motion of the hip and knee joint. There was no vascular or neurological deficit associated with the affected limp.

Plain radiography demonstrated a densely ossified mass in mid shaft with a cortical thickening of the right femur diaphysis (Figure 
1). Magnetic resonance imaging (MRI) revealed a tumour on the surface of the femoral diaphysis not involving the surrounding soft tissue (Figure 2). Subsequent PET scans of the chest and wholebody revealed no metastases or other bony abnormalities.

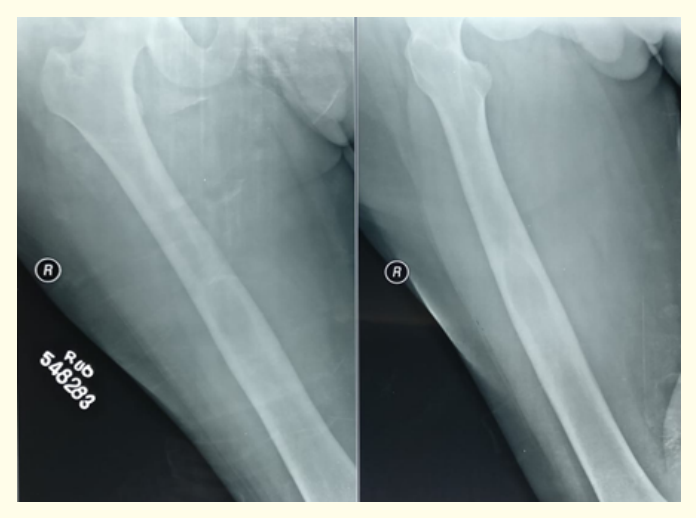

Figure 1: Anteroposterior and (B) lateral radiographs of the right femur demonstrate a densely ossified juxta cortical lesion.

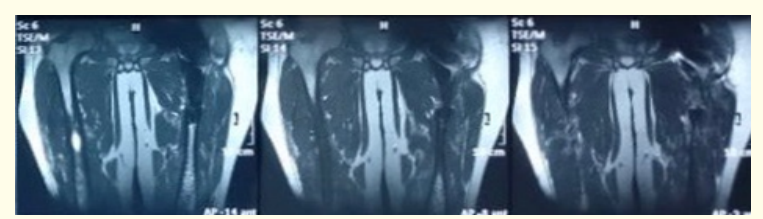

Figure 2: MR images demonstrating low-to-intermediate signal intensity tumour.

The surgical approach followed general principles of malignant bone tumour surgery. The patient was positioned supine, and the standard lateral approach to the femur was used, with the incision extending from the lateral knee joint line proximally to the lesser trochanter. The femur was osteotomized taking the safe margin (Figure $3 \mathrm{a}, \mathrm{b}$ ) and frozen section was send of the marrow at the site of proximal and distal resections were reviewed to ensure the margins were negative for tumour cells before reconstruction. The femoral diaphysis was then reconstructed using the Depuy modular intercalary endoprosthesis (Figure 4) which was augmented with locking LC-DCP to give rotational stability (Figure 5 and 6).

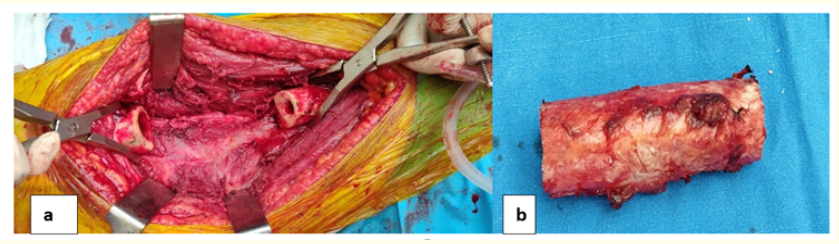

Figure 3: a. Femur was osteotomized taking the safe margin and; b. Segment of osteotomized femur.

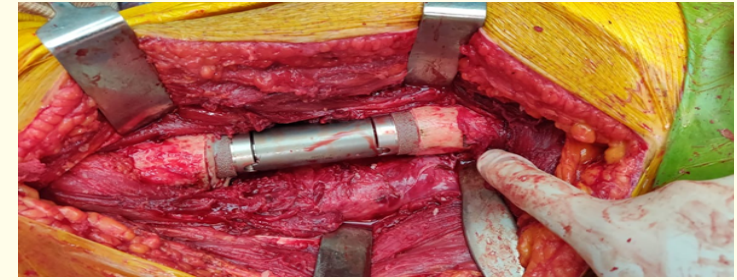

Figure 4: Intraoperative Picture of Intercalary Endoprosthesis in situ.

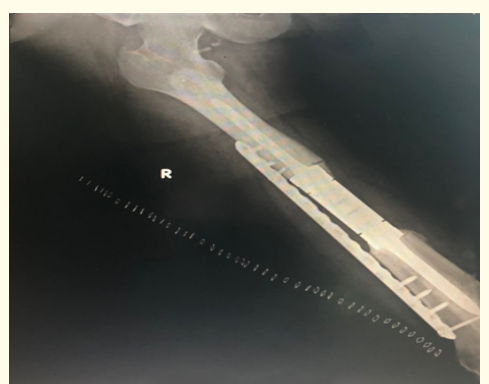

Figure 5: Immediate post. Op. Anteroposterior radiographs of the right femur with Intercalary endoprosthesis in situ augmented with Locking plate.

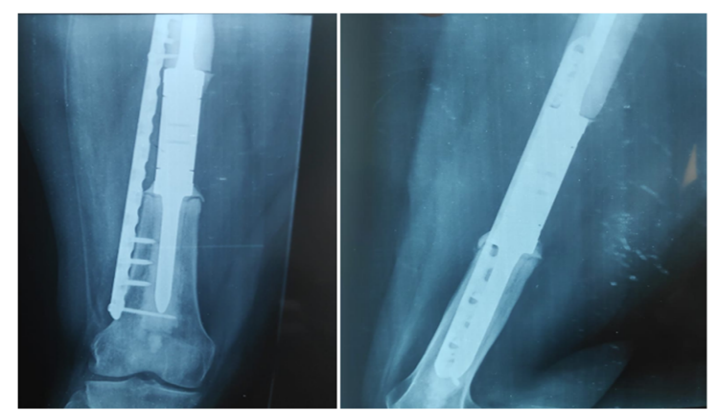

Figure 6: Showing Anteroposterior and (B) lateral radiographs of the right femur at 12 month follow up with very well fixed intercalary endoprosthesis.

The patient received prophylactic antibiotics (cefuroxime, $1.5 \mathrm{~g}$ ) twice a day for 3 days. Active physiotherapy commenced on the second day following the operation, and the patient was able to support full weight on the limb by the time of discharge. The patient recovered and returned to preoperative life. No local recurrence or metastasis was observed and the range of motion of the hip and knee was not restricted at the time of 12 months follow-up.

\section{Discussion}

Reconstruction of bone defects after diaphyseal femoral tumour resection is challenging. Ideally, reconstruction would provide stability, preservation and early motion of adjacent joints, and prolonged survival of the patients. Therefore, operative intervention is focused on achieving immediate pain relief and early function restoration within the limited life expectancy. 
Although biological reconstruction also preserves adjacent joints and restores function, the procedure is time-consuming, with a prolonged period of immobilization after surgery and a significant risk of non-union and/or fracture [13]. It has been suggested that non-union and infection rates are even higher in patients receiving adjuvant chemotherapy [14]. Immediate stability and pain relief provided by an intercalary prosthesis makes it a more reasonable alternative for patients with pathological fractures due to metastatic tumours of femoral diaphysis.

Length of bone resection and the remaining bone in prosthesis fixation are the most critical parameters affecting the application of intercalary prostheses. Benevenia., et al. recommended intercalary endoprosthesis as the only reconstruction method for patients with skeletal defects $\geq 4 \mathrm{~cm}$ in the humerus and $\geq 5 \mathrm{~cm}$ in the tibia and femur [15]. Ruggieri., et al. report that loosening is most common in the reconstruction of more than $10 \mathrm{~cm}$ length of bone resection [16]. Abudu., et al. suggest the shortest length of bone suitable for prosthesis fixation to be $5 \mathrm{~cm}$, but in this short segment, fixation is risky because of the possibility of early loosening [17]. Sewell., et al. suggest that fixation may be further enhanced by using extracortical plates.

Endoprosthesis replacements of the femoral diaphysis have demonstrated an acceptable postoperative complication rate, long-term survival and functional outcome, following resection of primary malignant bone tumors [14]. The complications encountered during this procedure include infection [18], aseptic loosening, mechanical failure, fracture either of the prosthesis or of adjacent bone [19], local recurrence and metastatic spread [14]. Compared with other reconstructive options, the use of a intercalary endoprosthesis for the reconstruction of the femoral diaphysis has some advantages. These include a short operating time and hospital stay, with a low incidence of early complications and reduced risk of disease transmission. Furthermore, it allows early patient mobility (without walking aids) and immediate commencement of post-operative adjuvant therapy. Patients are able to return to a more or less normal life much earlier [20].

In our case we used the modular intercalary implant. Our patient is now back to his normal life and doing all his day today activity. At 12 month follow up we did x-ray (Fig.6), PET CT scan which was not showing any signs of recurrence, metastasis and aseptic or septic loosening. We recommend the augmentation of locking plate with intercalary implant as it give added rotational stability to the construct.

\section{Conclusion}

Current literature support the use of intercalary endoprosthesis over other biological methods of treatment. As these intercalary implants helps in early recovery of the patients that is not possible in biological method of treatment. However, long-term follow studies are required to know the longevity of these implants and complication related to them.

\section{Conflict of Interest}

Nil.

\section{Bibliography}

1. Murphey MD., et al. "The many faces of osteosarcoma". Radiographics 17 (1997): 1205-1231.

2. Azura M., et al. "Parosteal osteosarcoma dedifferentiating into telan- giectatic osteosarcoma: Importance of lytic changes and fluid cavities at imaging". Skeletal Radiology 38 (2009): 685690.

3. Jelinek JS., et al. "Parosteal osteosarcoma: Value of MR imaging and CT in the prediction of histologic grade". Radiology 201 (1996) :837-842.

4. Park YK., et al. "Parosteal osteosarcoma of the scapula".Journal of Korean Medical Science 14 (1999): 586-588.

5. Subasi M., et al. "Unusual benign bone lesion simulating parosteal osteosarcoma". Journal of Orthopaedic Science 11 (2006): 529-532.

6. Partovi S., et al. "Low-grade parosteal osteosaracoma of the ulna with dedifferentiation into high-grade osteosarcoma". Skeletal Radiology 25 (1996) :497-500.

7. Dönmez FY., et al. "MRI findings in parosteal osteosarcoma: Correlation with histopathology". Diagnostic and Interventional Radiology 14 (2008) :142-152.

8. Chang DW., et al. "Use of a vascularized fibula bone flap and intercalary allograft for diaphyseal reconstruction after resection of primary extremity bone sarcomas". Plastic and Reconstructive Surgery 116 (2005):1918-1925.

9. Ogilvie CM., et al. "Long-term results for limb salvage with osteo- articular allograft reconstruction". Clinical Orthopaedics and Related Research 467 (2009): 2685-2690.

10. Krieg AH., et al. "Intercalary femoral reconstruction with extracorporeal irradiated autogenous bone graft in limb-salvage surgery". The Journal of Bone and Joint Surgery British 89 (2007): 366-371.

11. Tsuchiya H., et al. "Reconstruction using an autograft containing tumour treated by liquid nitrogen". The Journal of Bone and Joint Surgery British 87 (2005): 218-225.

12. Ahlmann ER., et al. "Intercalary endoprosthetic reconstruction for diaphyseal bone tumours". The Journal of Bone and Joint Surgery British 88 (2006): 1487-1491.

13. Damron TA., et al. "Intercalary spacers in the treatment of segmentally destructive diaphyseal humeral lesions in disseminated malignancies". Clinical Orthopaedics and Related Research 324 (1996): 233-243. 
14. Hanna SA., et al. "Femoral diaphyseal endoprosthetic reconstruction after segmental resection of primary bone tumours". The Journal of Bone and Joint Surgery British 92 (2010): 867874.

15. Zhao SC., et al. "Custom-made intercalary endoprosthetic reconstruction for a parosteal osteosarcoma of the femoral diaphysis: a case report”. Oncology Letters 10 (2015): 32793285.

16. Ruggieri P., et al. "Outcome of the intramedullary diaphyseal segmental defect fixation system for bone tumors". Journal of Surgical Oncology 104 (2011): 83-90.

17. Abudu A., et al. "The outcome and functional results of diaphyseal endoprostheses after tumour excision". The Journal of Bone and Joint Surgery British 78 (1996): 652-657.

18. Gosheger G., et al. "Endoprosthetic reconstruction in 250 patients with sarcoma". Clinical Orthopaedics and Related Research 450 (2006): 164-171.

19. Sewell MD., et al. "Total femoral endoprosthetic replacement following excision of bone tumours". The Journal of bone and joint surgery British 91 (2009): 1513-1520.

20. Muscolo DL., et al. "Intercalary femur and tibia segmental allografts provide an acceptable alternative in reconstructing tumor resections". Clinical Orthopaedics and Related Research 426 (2004): 97-102.

\section{Assets from publication with us}

- Prompt Acknowledgement after receiving the article

- Thorough Double blinded peer review

- Rapid Publication

- Issue of Publication Certificate

- High visibility of your Published work

Website: https://www.actascientific.com/

Submit Article: https://www.actascientific.com/submission.php

Email us: editor@actascientific.com

Contact us: +919182824667 